\title{
A Generalized Gradient Projection Filter Algorithm for Inequality Constrained Optimization
}

\author{
Wei Wang, ${ }^{1}$ Shaoli Hua, ${ }^{2}$ and Junjie Tang ${ }^{1}$ \\ ${ }^{1}$ Department of Mathematics, East China University of Science and Technology, Shanghai 200237, China \\ ${ }^{2}$ China UnionPay Merchant Services Co., Ltd., Shanghai 200135, China \\ Correspondence should be addressed to Wei Wang; wangwei@ecust.edu.cn
}

Received 27 May 2013; Revised 22 August 2013; Accepted 5 September 2013

Academic Editor: Igor Andrianov

Copyright (c) 2013 Wei Wang et al. This is an open access article distributed under the Creative Commons Attribution License, which permits unrestricted use, distribution, and reproduction in any medium, provided the original work is properly cited.

A generalized gradient projection filter algorithm for inequality constrained optimization is presented. It has three merits. The first is that the amount of computation is lower, since the gradient matrix only needs to be computed one time at each iterate. The second is that the paper uses the filter technique instead of any penalty function for constrained programming. The third is that the algorithm is of global convergence and locally superlinear convergence under some mild conditions.

\section{Introduction}

The optimal problems are often discovered in the field of management, engineering design, traffic transportation, national defence, and so on. The efficient algorithms for these problems are important. We will consider the following nonlinear inequality constrained optimization problem:

$$
\begin{array}{ll}
\min & f(x) \\
\text { s.t. } & c_{j}(x) \leq 0,
\end{array}
$$

where $I=\{1,2, \ldots, m\}$ and $x \in R^{n}$; assume that $f: R^{n} \rightarrow R$ and $c_{j}(j \in I): R^{n} \rightarrow R$ are continuously differentiable.

In 2002, Fletcher and Leyffer [1] had proposed a filter method for nonlinear inequality constrained optimization, which did not require any penalty function. The main idea is that a trial point is accepted if it improves either the objective function or the constraint violation. Fletcher et al. [2,3] and Gonzaga et al. [4] had proved that the method was of global convergence. More recently, this method has been extended by Wächter and Biegler [5,6] and Chin [7] to line search method and by Su [8] to the SQP method.

In this paper, we modify the method given by Wang et al. [9] and propose a generalized gradient projection filter algorithm for inequality constrained optimization with arbitrary initial point. It is organized as follows. In Section 2, we first review the filter method and some definitions of generalized gradient projection and then introduce an algorithm for problem (1). The convergence and the rate of convergence on the algorithm are discussed in Sections 3 and 4, respectively. In the last section, we shall list the numerical tests.

\section{Preliminaries and a Filter Algorithm}

Let $h(x)$ be a violation function; that is,

$$
h(x)=\max \left\{0, c_{j}(x), j \in I\right\} .
$$

Definition 1. A pair $\left(h\left(x_{k}\right), f\left(x_{k}\right)\right)$ obtained on iteration $k$ dominates another pair $\left(h\left(x_{l}\right), f\left(x_{l}\right)\right)$ if and only if $h\left(x_{k}\right) \leq$ $h\left(x_{l}\right)$ and $f\left(x_{k}\right) \leq f\left(x_{l}\right)$ hold.

Definition 2. A filter is a list of pairs $\left(h\left(x_{k}\right), f\left(x_{k}\right)\right)$ such that no pair dominates any other. A pair $\left(h\left(x_{k}\right), f\left(x_{k}\right)\right)$ is said to be acceptable for the filter if it is not dominated by any point in the filter.

We use $F^{(k)}$ to denote the set of iterations indices $j(j<$ $k$ ) such that $\left(h\left(x_{j}\right), f\left(x_{j}\right)\right)$ is an entry in the current filter. A point $x$ is said to be "acceptable for the filter" if and only if

$$
h(x) \leq\left(1-\alpha^{2} \eta\right) h\left(x_{j}\right) \quad \text { or } \quad f(x) \leq f\left(x_{j}\right)-\gamma h\left(x_{j}\right)
$$


holds for all $j \in F^{(k)}$, where $\gamma, \eta \in(0,1)$ is close to zero and $\alpha$ is the step size. We may also "update the filter" which means that the pair $(h(x), f(x))$ is added to the list of pairs in the filter, and any pairs in the filter that are dominated by $(h(x), f(x))$ are removed.

However, relying solely on this criterion would result in convergence to a feasible but nonoptimal point. In order to prevent this, we employ the following sufficient reduction criterion.

We denote $\Delta f_{k}=f\left(x_{k}\right)-f\left(x_{k}+\alpha d_{k}\right)$ and $\Delta l_{k}=$ $-\alpha \nabla f\left(x_{k}\right)^{T} d_{k}$ as actual reduction and linear reduction, respectively, at $f\left(x_{k}\right)$. The sufficient reduction condition for $f\left(x_{k}\right)$ takes the form

$$
\Delta l_{k} \geq 0, \quad \Delta f_{k} \geq \sigma \Delta l_{k},
$$

where $\sigma \in(0,1 / 2)$ is a preassigned parameter.

At the current iterate $x_{k}$, define that $J\left(x_{k}\right)=\{j \in I$ : $\left.\epsilon \leq c_{j}\left(x_{k}\right)-h\left(x_{k}\right) \leq 0\right\}, A_{k}=\left(\nabla c_{j}\left(x_{k}\right), j \in J\left(x_{k}\right)\right)$, and $c_{J_{k}}=\left(c_{j}\left(x_{k}\right), j \in J\left(x_{k}\right)\right)^{T}$, and then

$$
\begin{gathered}
d_{k}^{0}=-P_{k} \nabla f\left(x_{k}\right)-B_{k}^{T} c_{J_{k}}, \\
\lambda_{k}=-B_{k} \nabla f\left(x_{k}\right)+\left(A_{k}^{T} H_{k} A_{k}\right)^{-1} c_{J_{k}}=\lambda_{k}^{1}+\lambda_{k}^{2},
\end{gathered}
$$

where $H_{k}$ is a given symmetric positive definite matrix, $\lambda_{k}^{1}=$ $-B_{k} \nabla f\left(x_{k}\right), \lambda_{k}^{2}=\left(A_{k}^{T} H_{k} A_{k}\right)^{-1} c_{J_{k}}, B_{k}=\left(A_{k}^{T} H_{k} A_{k}\right)^{-1} A_{k}^{T} H_{k}$, and $P_{k}=H_{k}-H_{k} A_{k} B_{k}$.

Let $U_{k}=\left(u_{k_{j}}, j \in J\left(x_{k}\right)^{T}\right)$, where $u_{k_{j}}=\left\{\begin{array}{cc}\lambda_{k_{j}}^{1} & \lambda_{k_{j}}^{1}<0 \\ 0 & \lambda_{k_{j}}^{1} \geq 0\end{array}\right.$. Set $d_{k}^{1}=-P_{k} \nabla f\left(x_{k}\right)+B_{k}^{T} U_{k}$ and $d_{k}^{2}=-P_{k} \nabla f\left(x_{k}\right)+B_{k}^{T}\left\|d_{k}^{1}\right\| e$, where $e=(1, \ldots, 1)^{T}$. Then

$$
d_{k}=\left(1-\rho_{k}\right) d_{k}^{1}+\rho_{k} d_{k}^{2},
$$

where $\rho_{k}=\max \left\{\rho \in(0,1]: \nabla f\left(x_{k}\right)^{T}\left((1-\rho) d_{k}^{1}+\rho d_{k}^{2}\right) \leq\right.$ $\left.\theta \nabla f\left(x_{k}\right)^{T} d_{k}^{1}\right\}, \theta \in(1 / 2,1)$. We use correction direction $d_{k}$ if a trial point has been rejected.

The following is the algorithm.

\section{Algorithm}

(S0) Given start point $x_{0} \in R^{n}, \epsilon_{0}, \epsilon_{1}>0, \mu=h\left(x_{0}\right), \eta, \gamma \in$ $(0,1)$, and $\beta, t, \sigma \in(0,1 / 2)$. Initialize the filter $\Phi_{0}=$ $\left\{(\mu,+\infty) \in R^{2}\right\}$ and $F^{(0)}=\emptyset$. Set $k=0$.

(S1) Inner loop A:

(S1.1) set $i=0$ and $\epsilon_{k_{i}}=\epsilon_{0}$;

(S1.2) if $\operatorname{det}\left(A_{k_{i}}^{T} A_{k_{i}}\right) \geq \epsilon_{k_{i}}$, where $A_{k_{i}}=\left(\nabla c_{j}\left(x_{k}\right): j \epsilon\right.$ $\left.J_{k_{i}}\right)$ and $J_{k_{i}}=\left\{j \in I: \epsilon_{k_{i}} \leq c_{j}\left(x_{k}\right)-h\left(x_{k}\right) \leq 0\right\}$, then set $J\left(x_{k}\right)=J_{k_{i}}, A_{k}=A_{k_{i}}$, and $\epsilon_{k}=\epsilon_{k_{i}}$, and go to $S 2$;

(S1.3) let $i=i+1, \epsilon_{k_{i}}=\epsilon_{k_{i-1}} / 2$, and go to S1.2.

(S2) Compute $d_{k}^{0}, \lambda_{k}$ by (5). If $d_{k}^{0}=0$ and $\lambda_{k} \geq 0$, then stop.

(S3) Test direction $d_{k}^{0}$ :
(S3.1) if $\lambda_{k j} \geq \epsilon_{1}$, and $x_{k}+d_{k}^{0}$ is acceptable for the filter, then go to S3.2; otherwise, go to S4;

(S3.2) if $h\left(x_{k}\right)>0$, let $x_{k+1}=x_{k}+d_{k}^{0}$, and go to S7; otherwise, go to S3.3;

(S3.3) if $x_{k}+d_{k}^{0}$ satisfies the sufficient reduction condition (4), then let $x_{k+1}=x_{k}+d_{k}^{0}$, and go to S7; otherwise, go to S4.

(S4) Compute $d_{k}$ by (6) and set $\alpha=1$.

(S5) Inner loop B:

(S5.1) if $x_{k}+\alpha d_{k}$ is acceptable for the filter, go to S5.2; otherwise, go to S5.3;

(S5.2) if $\Delta f_{k}<\sigma \Delta l_{k}$, go to S5.3; otherwise, go to S6;

(S5.3) set $\alpha=t \alpha$, and go to S5.1.

(S6) Set $\alpha_{k}=\alpha$ and $x_{k+1}=x_{k}+\alpha d_{k}$.

(S7) Update filter $F^{(k)}$ to $F^{(k+1)}$. Update $H_{k}$ to $H_{k+1}$ by a quasi-Newton method. Set $k=k+1$, and back to S1.

\section{Global Convergence of the Algorithm}

In this section, we assume that the following conditions hold.

(A1) $\left\{\nabla c_{j}(x), j \in J(x)\right\}$ is linearly independent of any $x \in$ $R^{n}$.

(A2) For any $k$ and $d$, $a\|d\|^{2} \leq d^{T} H_{k}^{-1} d \leq b\|d\|^{2}$ holds, where $0<a \leq b$ are constants.

(A3) Sequence $\left\{x_{k}\right\}$ generated by the algorithm remains in a closed, bounded subset $\Omega \subset R^{n}$.

(A4) $f(x)$ and $c_{i}(x)(i=1,2, \ldots, m)$ are twice differentiable in $\Omega$; that is, $M_{\min }^{f} \leq \lambda\left(\nabla^{2} f(x)\right) \leq M_{\max }^{f}, M_{\min }^{c} \leq$ $\lambda\left(\nabla^{2} c_{i}(x)\right) \leq M_{\max }^{c}$

Similar to [9], the following theorem and lemma hold.

Theorem 3. If $d_{k}^{0}=0$ and $\lambda_{k} \geq 0$ hold, then $x_{k}$ is a KKT point of problem (1).

Lemma 4. Consider

$$
d_{k}^{0}=0, \quad \lambda_{k} \geq 0 \Longleftrightarrow d_{k}^{1}=0 .
$$

According to [8], the following lemma holds.

Lemma 5. The inner loop A will terminate in finite times.

Lemma 6. If $x_{k}$ is not a KKT point of problem (1), there must exist $\nabla f\left(x_{k}\right)^{T} d_{k}<0$ and $\nabla c_{j}\left(x_{k}\right)^{T} d_{k}<0, j \in J\left(x_{k}\right)$.

Proof. Since $x_{k}$ is not a KKT point, we have either $d_{k}^{0} \neq 0$ or $j \in J\left(x_{k}\right)$ such that $\lambda_{k j}^{1}<0$. Thus

$$
\begin{aligned}
\nabla f\left(x_{k}\right)^{T} d_{k} & \leq \theta \nabla f\left(x_{k}\right)^{T} d_{k}^{1} \\
& \leq \theta\left[-\left(d_{k}^{0}\right)^{T} H_{k}^{-1} d_{k}^{0}-\sum_{\lambda_{k_{j}}^{1}<0}\left(\lambda_{k_{j}}^{1}\right)^{2}\right]<0
\end{aligned}
$$


holds. From Lemma 4 , we know that $d_{k}^{1} \neq 0$. Therefore

$$
A_{J_{k}}^{T} d_{k}^{1}=U_{k} \leq 0, \quad A_{J_{k}}^{T} d_{k}^{2}=-\left\|d_{k}^{1}\right\| e<0 .
$$

That is, $\nabla c_{j}\left(x_{k}\right)^{T} d_{k}<0\left(j \in J\left(x_{k}\right)\right)$ hold.

Lemma 7. Let $x^{\infty}$ be the cluster point of $\left\{x_{k}\right\}$ generated by algorithm. If $x^{\infty}$ is not the KKT point of problem (1), there exists $\bar{\alpha}>0$, such that $\Delta f_{k} \geq \sigma \Delta l_{k}$ holds when $\alpha \leq \bar{\alpha}$.

Proof. From the definition of $\rho$ and the assumption (A2), we have

$$
\begin{aligned}
\Delta l_{k} & \geq-\alpha \theta \nabla f\left(x_{k}\right)^{T} d_{k}^{1} \\
& =-\alpha \theta\left[-\left(d_{k}^{0}\right)^{T} H_{k}^{-1} d_{k}^{0}-\sum_{\lambda_{k_{j}}^{1}<0}\left(\lambda_{k_{j}}^{1}\right)^{2}\right] \\
& \geq \alpha \theta\left(d_{k}^{0}\right)^{T} H_{k}^{-1} d_{k}^{0} \geq \frac{\alpha \theta a}{2}\left\|d_{k}^{0}\right\|^{2} .
\end{aligned}
$$

Since

$$
\begin{aligned}
\left|\Delta f_{k}-\Delta l_{k}\right| & =\left|f\left(x_{k}\right)-f\left(x_{k}+\alpha d_{k}\right)+\alpha \nabla f\left(x_{k}\right)^{T} d_{k}\right| \\
& \leq \frac{1}{2} \alpha^{2} M_{\max }^{f}\left\|d_{k}\right\|^{2}
\end{aligned}
$$

we have

$$
\left|\frac{\Delta f_{k}-\Delta l_{k}}{\Delta l_{k}}\right| \leq \frac{(1 / 2) \alpha^{2} M_{\max }^{f}\left\|d_{k}\right\|^{2}}{(\alpha \theta a / 2)\left\|d_{k}^{0}\right\|^{2}}=\frac{\alpha M_{\max }^{f}\left\|d_{k}\right\|^{2}}{\theta a\left\|d_{k}^{0}\right\|^{2}}
$$

It is easy to learn that $\Delta f_{k} \geq \sigma \Delta l_{k}$ holds when $\alpha \leq \bar{\alpha}=(1-$ $\sigma) \theta a\left\|d_{k}^{0}\right\|^{2} / M_{\max }^{f}\left\|d_{k}\right\|^{2}$.

Lemma 8. The inner loop B will end in finite times.

Proof. From Lemma 7, we have that $\Delta f_{k} \geq \sigma \Delta l_{k}$ holds when $\alpha \leq \bar{\alpha}$. By contradiction, if the conclusion is false, then the algorithm will run infinitely between S5.1 and S5.3, so we have $\alpha \rightarrow 0$ and $x_{k}+\alpha d_{k}$ not acceptable for the filter. We consider it in the following two cases.

Case $1\left(h\left(x_{k}\right)=0\right)$. From Lemma 6, we have $\nabla f\left(x_{k}\right)^{T} d_{k}<0$ and $\nabla c_{j}\left(x_{k}\right)^{T} d_{k}<0, j \in J\left(x_{k}\right)$. So when

$\alpha \leq \min \left\{\frac{-\nabla f\left(x_{k}\right)^{T} d_{k}}{(1 / 2) M_{\max }^{f}\left\|d_{k}\right\|^{2}}, \min _{j \in J\left(x_{k}\right)}\left\{\frac{-\nabla c_{j}\left(x_{k}\right)^{T} d_{k}}{(1 / 2) M_{\max }^{c}\left\|d_{k}\right\|^{2}}\right\}\right\}$, it is easy to get that

$$
\begin{aligned}
f\left(x_{k}+\alpha d_{k}\right) \leq & f\left(x_{k}\right)+\alpha \nabla f\left(x_{k}\right)^{T} d_{k} \\
& +\frac{1}{2} \alpha^{2} M_{\max }^{f}\left\|d_{k}\right\|^{2} \leq f\left(x_{k}\right) \\
= & f\left(x_{k}\right)-\gamma h\left(x_{k}\right), \\
h\left(x_{k}+\alpha d_{k}\right) \leq & \max \left\{0, c_{j}\left(x_{k}\right)+\alpha \nabla c_{j}\left(x_{k}\right)^{T} d_{k}\right. \\
& \left.+\frac{1}{2} \alpha^{2} M_{\max }^{c}\left\|d_{k}\right\|^{2}\right\} \\
\leq & \max \left\{0, c_{j}\left(x_{k}\right)\right\} \\
= & \left(1-\alpha^{2} \eta\right) \max \left\{0, c_{j}\left(x_{k}\right)\right\} \\
= & \left(1-\alpha^{2} \eta\right) h\left(x_{k}\right) .
\end{aligned}
$$

It proves that $x_{k}+\alpha d_{k}$ is acceptable for the filter.

Case $2\left(h\left(x_{k}\right)>0\right)$. Similarly, when

$$
\alpha \leq \min _{j \in J\left(x_{k}\right)}\left\{\frac{-\nabla c_{j}\left(x_{k}\right)^{T} d_{k}}{(1 / 2) M_{\max }^{c}\left\|d_{k}\right\|^{2}+\eta c_{j}\left(x_{k}\right)}\right\},
$$

it is easy to learn that

$$
\begin{aligned}
& h\left(x_{k}+\alpha d_{k}\right) \\
& \quad \leq \max \left\{0, c_{j}\left(x_{k}\right)+\alpha \nabla c_{j}\left(x_{k}\right)^{T} d_{k}+\frac{1}{2} \alpha^{2} M_{\max }^{c}\left\|d_{k}\right\|^{2}\right\} \\
& \quad \leq\left(1-\alpha^{2} \eta\right) h\left(x_{k}\right) .
\end{aligned}
$$

Since $x_{k}$ is acceptable for the filter, so for all $j \in F^{(k-1)}, h\left(x_{k}\right) \leq$ $h\left(x_{j}\right)$ or $f\left(x_{k}\right) \leq f\left(x_{j}\right)-\gamma h\left(x_{j}\right)$ holds. From $x_{k}+\alpha d_{k}$ that is not acceptable for the filter, we have

$$
\begin{aligned}
& h\left(x_{k}+\alpha d_{k}\right)>\left(1-\alpha^{2} \eta\right) h\left(x_{j}\right), \\
& f\left(x_{k}+\alpha d_{k}\right)>f\left(x_{j}\right)-\gamma h\left(x_{j}\right)
\end{aligned}
$$

hold. If $h\left(x_{k}\right) \leq h\left(x_{j}\right)$, then

$$
h\left(x_{k}+\alpha d_{k}\right) \leq\left(1-\alpha^{2} \eta\right) h\left(x_{k}\right) \leq\left(1-\alpha^{2} \eta\right) h\left(x_{j}\right),
$$

which contradicts (17). If $f\left(x_{k}\right) \leq f\left(x_{j}\right)-\gamma h\left(x_{j}\right)$, then when $\alpha \leq-\nabla f\left(x_{k}\right)^{T} d_{k} /(1 / 2) M_{\max }^{f}\left\|d_{k}\right\|^{2}$, it is easy to learn that

$$
\begin{aligned}
f\left(x_{k}+\alpha d_{k}\right) \leq & f\left(x_{k}\right)+\alpha \nabla f\left(x_{k}\right)^{T} d_{k} \\
& +\frac{1}{2} \alpha^{2} M_{\max }^{f}\left\|d_{k}\right\|^{2} \leq f\left(x_{k}\right) \\
\leq & f\left(x_{j}\right)-\gamma h\left(x_{j}\right),
\end{aligned}
$$

which contradicts (18).

Based on the above analysis, we can see that the claim holds. 
By the above statement, we can see that the algorithm is implementable. Now we turn on to prove the global convergence of the algorithm.

Theorem 9. Let the assumptions hold and $M_{\text {min }}^{f}>0$. Suppose $x^{\infty}$ be the cluster point of $\left\{x_{k}\right\}$ generated by algorithm. There exist two possible cases. (i) The iteration terminates at a KKT point. (ii) Any accumulation point of $\left\{x_{k}\right\}$ is a KKT point.

Proof. we only need to proof case (ii). Since $x^{\infty}$ is the cluster point generated by algorithm, let $\left\{x_{k}\right\}_{k \in K}$ be any thinner subsequences converging to $x^{\infty}$.

We will first show that $x^{\infty}$ is a feasible point. Assume that $h\left(x_{k}\right) \rightarrow h\left(x^{\infty}\right)>0$ for $k \in K$. Let $i$ and $j$ be any two adjacent indices in $K$ where $i<j$. If $h\left(x^{\infty}\right)>0$, then there exists $k^{\prime} \in K$ such that for all $i \geq k^{\prime}$ and because $x_{j}$ is acceptable to the filter, we have

$$
f\left(x_{j}\right) \leq f\left(x_{i}\right)-\gamma h\left(x_{i}\right) .
$$

Since $\left\{f\left(x_{k}\right)\right\}_{k \in K}$ is a monotonically decreasing subsequence for $k \geq k^{\prime}$ and is bounded below, therefore for $i, j \in K, i, j \geq$ $k^{\prime}$, and $i<j$,

$$
\sum_{i, j \in K} \Delta f_{i j}=\sum_{i, j \in K}\left(f\left(x_{i}\right)-f\left(x_{j}\right)\right)
$$

is bounded above. However, since $f\left(x_{j}\right) \leq f\left(x_{i}\right)-\gamma h\left(x_{i}\right)$, therefore by summing over all indices $i, j \in K, i, j \geq k^{\prime}$, and $i<j$,

$$
\sum_{i, j \in K} \Delta f_{i j} \geq \gamma \sum_{i \in K} h\left(x_{i}\right) \longrightarrow+\infty
$$

which contradicts the fact that $\sum_{i, j \in K} \Delta f_{i j}$ is bounded above. Thus $h\left(x^{\infty}\right)=0$, hence $x^{\infty}$ is feasible.

Next we need to show that $x^{\infty}$ is a KKT point. By the construction of algorithm, there are two cases: one generates the sequence $\left\{x_{k}\right\}$ from $x_{k+1}=x_{k}+d_{k}^{0}$, and the other generates it from $x_{k+1}=x_{k}+\alpha d_{k}$. We prove that claim according to the two cases.

Case 1. Suppose that there are infinite points gotten by $x_{k+1}=$ $x_{k}+d_{k}^{0}$. Since $\Delta f_{k} \geq \sigma \Delta l_{k}$, we have

$$
\begin{aligned}
f\left(x_{k}\right)-f\left(x_{k}+d_{k}^{0}\right)= & -\nabla f\left(x_{k}\right)^{T} d_{k}^{0} \\
& -\frac{1}{2}\left(d_{k}^{0}\right)^{T} \nabla^{2} f(y) d_{k}^{0} \\
\geq & -\sigma \nabla f\left(x_{k}\right)^{T} d_{k}^{0} .
\end{aligned}
$$

Thus $\nabla f\left(x_{k}\right)^{T} d_{k}^{0} \leq-(1 / 2)\left(d_{k}^{0}\right)^{T} \nabla^{2} f(y) d_{k}^{0} /(1-\sigma)$ holds. Since $f$ is bounded below, then

$$
\begin{aligned}
+\infty & >\sum_{k=0}^{\infty} f\left(x_{k}\right)-f\left(x_{k+1}\right) \geq-\sum_{k=0}^{\infty} \nabla f\left(x_{k}\right)^{T} d_{k}^{0} \\
& \geq \frac{1}{2} \sum_{k=0}^{\infty} \frac{\left(d_{k}^{0}\right)^{T} \nabla^{2} f(y) d_{k}^{0}}{1-\sigma} \geq \frac{M_{\min }^{f}}{2(1-\sigma)} \sum_{k=0}^{\infty}\left\|d_{k}^{0}\right\|^{2} .
\end{aligned}
$$

Thus $\sum_{k=0}^{\infty}\left\|d_{k}^{0}\right\|^{2}<+\infty$, which means $\left\|d_{k}^{0}\right\| \rightarrow 0$. Since $x^{\infty}$ is a feasible point, $x^{\infty}$ is a KKT point.

Case 2. Suppose that there are infinite points gotten by $x_{k+1}=$ $x_{k}+\alpha d_{k}$. Since $\Delta f_{k} \geq \sigma \Delta l_{k}$, we have

$$
\begin{aligned}
0 & =\lim _{k \rightarrow \infty} f\left(x_{k}\right)-f\left(x_{k}+\alpha d_{k}\right) \\
& \geq-\lim _{k \rightarrow \infty} \sigma \alpha \nabla f\left(x_{k}\right)^{T} d_{k} \geq-\lim _{k \rightarrow \infty} \bar{\alpha} \sigma \nabla f\left(x_{k}\right)^{T} d_{k} \geq 0,
\end{aligned}
$$

which means that $\nabla f\left(x_{k}\right)^{T} d_{k} \rightarrow 0$. Since

$$
\begin{aligned}
\nabla f\left(x_{k}\right)^{T} d_{k} & \leq \theta \nabla f\left(x_{k}\right)^{T} d_{k}^{1} \\
& \leq \theta\left[-\left(d_{k}^{0}\right)^{T} H_{k}^{-1} d_{k}^{0}-\sum_{\lambda_{k j}^{1}<0}\left(\lambda_{k j}^{1}\right)^{2}\right]<0
\end{aligned}
$$

we have $\left\|d_{k}^{0}\right\| \rightarrow 0$ and $\lambda_{k j} \geq 0$, and since $x^{\infty}$ is a feasible point, $x^{\infty}$ is a KKT point.

Combined Case 1 and Case 2, we can see that the claim holds.

\section{The Rate of Convergence}

In this section, we discuss the convergent rate of the algorithm. We need the following strong assumptions.

(A5) The second-order sufficiently conditions hold, that is, $d^{T} \nabla_{x x}^{2} L\left(x^{\infty}, \lambda^{\infty}\right) d$, for all $d \in \operatorname{ker} \nabla c_{\widehat{J}\left(x^{\infty}\right)} \mid$ $\{0\}$, where $L(x, \lambda)=f(x)+\lambda^{T} c(x), c(x)=$ $\left(c_{1}(x), \ldots, c_{m}(x)\right)^{T}, \widehat{J}\left(x^{\infty}\right)=\left\{j \in J\left(x^{\infty}\right):\left(\lambda^{\infty}\right)_{j}>0\right\}$, and $\left(x^{\infty}, \lambda^{\infty}\right)$ is the KKT pair of problem (1).

(A6) Consider $\left\|\left(H_{k}^{-1}-\nabla_{x x}^{2} L\left(x^{\infty}, \lambda^{\infty}\right)\right) d_{k}^{0}\right\|=o\left(\left\|d_{k}^{0}\right\|\right)$.

Theorem 10. Suppose that assumptions (A1)-(A6) hold; then $x_{k+1}=x_{k}+d_{k}^{0}$ for large enough $k$. Therefore the algorithm is superlinearly convergent.

Proof. Suppose that $x_{k}$ is acceptable for the filter; we will show that for large enough $k, x_{k+1}=x_{k}+d_{k}^{0}$ is acceptable for the filter and satisfies the sufficient reduction condition.

First we need to prove that $x_{k+1}=x_{k}+d_{k}^{0}$ is acceptable for the filter. If $h\left(x_{k}+d_{k}^{0}\right) \leq(1-\eta) h\left(x_{k}\right)$, then $x_{k+1}=x_{k}+d_{k}^{0}$ is already acceptable for the filter. Else we need to show that $f\left(x_{k}+d_{k}^{0}\right) \leq f\left(x_{k}\right)-\gamma h\left(x_{k}\right)$. Let $s_{k}=f\left(x_{k}+d_{k}^{0}\right)-f\left(x_{k}\right)+$ $\gamma h\left(x_{k}\right)$; it holds that

$$
\begin{aligned}
s_{k} \leq & \nabla f\left(x_{k}\right)^{T} d_{k}^{0}+\frac{1}{2}\left(d_{k}^{0}\right)^{T} \nabla^{2} f\left(x_{k}\right) d_{k}^{0} \\
& +\frac{\gamma h\left(x_{k}+d_{k}^{0}\right)}{1-\eta}+o\left(\left\|d_{k}^{0}\right\|^{2}\right) \leq \nabla f\left(x_{k}\right)^{T} d_{k}^{0} \\
& +\frac{1}{2}\left(d_{k}^{0}\right)^{T} \nabla^{2} f\left(x_{k}\right) d_{k}^{0} \\
& +\frac{\gamma}{2(1-\eta)} \sum_{j=1}^{m}\left(d_{k}^{0}\right)^{T} \nabla^{2} c_{j}\left(x_{k}\right) d_{k}^{0}+o\left(\left\|d_{k}^{0}\right\|^{2}\right) .
\end{aligned}
$$


From $\nabla f\left(x_{k}\right)^{T} d_{k}^{0}=\lambda_{k}^{T} c_{J_{k}}-\left(d_{k}^{0}\right)^{T} H_{k}^{-1} d_{k}^{0}$, we have

$$
\begin{aligned}
s_{k} \leq & \lambda_{k}^{T} c_{J_{k}}-\left(d_{k}^{0}\right)^{T} H_{k}^{-1} d_{k}^{0}+\frac{1}{2}\left(d_{k}^{0}\right)^{T} \nabla^{2} f\left(x_{k}\right) d_{k}^{0} \\
& +\frac{\gamma}{2(1-\eta)} \sum_{j=1}^{m}\left(d_{k}^{0}\right)^{T} \nabla^{2} c_{j}\left(x_{k}\right) d_{k}^{0}+o\left(\left\|d_{k}^{0}\right\|^{2}\right) .
\end{aligned}
$$

Since $\lambda_{k j} \geq \epsilon_{1}$, set $\epsilon_{1}=\gamma /(1-\eta)$, and then

$$
\begin{aligned}
s_{k} \leq & \lambda_{k}^{T} c_{J_{k}}-\left(d_{k}^{0}\right)^{T} H_{k}^{-1} d_{k}^{0}+\frac{1}{2}\left(d_{k}^{0}\right)^{T} \nabla^{2} f\left(x_{k}\right) d_{k}^{0} \\
+ & \frac{1}{2} \sum_{j=1}^{m} \lambda_{k j}\left(d_{k}^{0}\right)^{T} \nabla^{2} c_{j}\left(x_{k}\right) d_{k}^{0}+o\left(\left\|d_{k}^{0}\right\|^{2}\right) \\
= & -\left(d_{k}^{0}\right)^{T} H_{k}^{-1} d_{k}^{0}+\lambda_{k}^{T} c_{J_{k}} \\
& +\frac{1}{2}\left(d_{k}^{0}\right)^{T} \nabla_{x x}^{2} L\left(x_{k}, \lambda_{k}\right) d_{k}^{0}+o\left(\left\|d_{k}^{0}\right\|^{2}\right) .
\end{aligned}
$$

According to $x_{k} \rightarrow x^{\infty}, \lambda_{k} \rightarrow \lambda^{\infty} \geq 0$, and $c_{j}\left(x_{k}\right) \rightarrow$ $c_{j}\left(x^{\infty}\right) \leq 0$ and assumptions (A2), (A3), and (A5), then

$$
\begin{aligned}
s_{k} \leq & -\frac{a}{2}\left\|d_{k}^{0}\right\|^{2} \\
& +\frac{1}{2}\left(d_{k}^{0}\right)^{T}\left(\nabla_{x x}^{2} L\left(x_{k}, \lambda_{k}\right)-\nabla_{x x}^{2} L\left(x^{\infty}, \lambda^{\infty}\right)\right) d_{k}^{0} \\
& +o\left(\left\|d_{k}^{0}\right\|^{2}\right)+\frac{1}{2}\left(d_{k}^{0}\right)^{T}\left(\nabla_{x x}^{2} L\left(x^{\infty}, \lambda^{\infty}\right)-H_{k}^{-1}\right) d_{k}^{0} \\
\leq & -\frac{a}{2}\left\|d_{k}^{0}\right\|^{2}+o\left(\left\|d_{k}^{0}\right\|^{2}\right) \leq 0 .
\end{aligned}
$$

Hence, for large enough $k, x_{k+1}=x_{k}+d_{k}^{0}$ is acceptable for the filter.

Now we are going to show that when $k$ is large enough, $x_{k+1}=x_{k}+d_{k}^{0}$ satisfies the sufficient reduction condition $\Delta f_{k} \geq \sigma \Delta l_{k}$. Let $t_{k}=f\left(x_{k}\right)-f\left(x_{k}+d_{k}^{0}\right)-\sigma \Delta l_{k}$; then we have

$$
\begin{aligned}
t_{k}= & (\sigma-1)\left(\lambda_{k}^{T} c_{J_{k}}-\left(d_{k}^{0}\right)^{T} H_{k}^{-1} d_{k}^{0}\right) \\
& -\frac{1}{2}\left(d_{k}^{0}\right)^{T} \nabla^{2} f\left(x_{k}\right) d_{k}^{0}-o\left(\left\|d_{k}^{0}\right\|^{2}\right) \\
\geq & (\sigma-1)\left(\lambda_{k}^{T} c_{J_{k}}-\left(d_{k}^{0}\right)^{T} H_{k}^{-1} d_{k}^{0}\right) \\
& -\frac{1}{2}\left(d_{k}^{0}\right)^{T} \nabla_{x x}^{2} L\left(x_{k}, \lambda_{k}\right) d_{k}^{0}-o\left(\left\|d_{k}^{0}\right\|^{2}\right) .
\end{aligned}
$$

Since $c_{j}\left(x_{k}\right) \rightarrow c_{j}\left(x^{\infty}\right) \leq 0$ and assumptions (A3), and (A5), then

$$
\begin{aligned}
t_{k} \geq & (\sigma-1) \lambda_{k}^{T} \mathcal{C}_{J_{k}}-\left(\sigma-\frac{1}{2}\right)\left(d_{k}^{0}\right)^{T} H_{k}^{-1} d_{k}^{0} \\
& -\frac{1}{2}\left(d_{k}^{0}\right)^{T}\left(\nabla_{x x}^{2} L\left(x_{k}, \lambda_{k}\right)-\nabla_{x x}^{2} L\left(x^{\infty}, \lambda^{\infty}\right)\right) d_{k}^{0} \\
& -\frac{1}{2}\left(d_{k}^{0}\right)^{T}\left(\nabla_{x x}^{2} L\left(x^{\infty}, \lambda^{\infty}\right)-H_{k}^{-1}\right) d_{k}^{0}-o\left(\left\|d_{k}^{0}\right\|^{2}\right) \\
\geq & \frac{a}{2}\left(\frac{1}{2}-\sigma\right)\left\|d_{k}^{0}\right\|^{2}-o\left(\left\|d_{k}^{0}\right\|^{2}\right) \geq 0 .
\end{aligned}
$$

Hence, for large enough $k, x_{k+1}=x_{k}+d_{k}^{0}$ satisfies the sufficient reduction condition.

Based on Theorem 10, we can see, when $k$ is large enough that the algorithm will implement the Newton steps and will not change; thus the algorithm is superlinearly convergent.

\section{Numerical Test}

In this section, we give some numerical results according to our algorithm. We update the matrix $H_{k}$ by BFGS formulation and the algorithm parameters are set as $H_{0}=I \epsilon$ $R^{n \times n}, \gamma=0.1, \eta=0.1$, and $\sigma=0.01$.

Example 11. One has

$$
\begin{aligned}
& \min f(x)=0.1\left\{0.44 \frac{x_{1}^{3}}{x_{2}^{2}}+\frac{10}{x_{1}}+0.592 \frac{x_{1}}{x_{2}^{3}}\right\} \\
& \text { s.t. } \quad-1+8.62 \frac{x_{2}^{3}}{x_{1}} \leq 0,
\end{aligned}
$$

where $x_{0}=(2.5,2.5), x^{\infty}=(1.2867,0.5305)$, and iterate $=$ 16.

Example 12 (see [8]). Consider

$$
\begin{array}{ll}
\min & f(x)=x_{1}^{2}+x_{2}^{2}+x_{3}^{2}+x_{4}^{2} \\
\text { s.t. } & 6-x_{1}^{2}-x_{2}^{2}-x_{3}^{2}-x_{4}^{2} \leq 0
\end{array}
$$

where $x_{0}=(2,2,2,2), x^{\infty}$ $(1.2247,1.2247,1.2247,1.2247)$, and iterate $=14$.

Example 13 (see [10]). One has

$$
\begin{array}{ll}
\min f(x)= & -50\left(x_{1}^{2}+x_{2}^{2}+x_{3}^{2}+x_{4}^{2}+x_{5}^{2}\right) \\
& -10.5 x_{1}-7.5 x_{2}-3.5 x_{3}-2.5 x_{4} \\
& -1.5 x_{5}-10 x_{6} \\
\text { s.t. } \quad 6 x_{1}+3 x_{2}+3 x_{3}+2 x_{4}+x_{5} \leq 6.5 & \\
& 10 x_{1}+10 x_{3}+x_{6} \leq 20 \\
& 0 \leq x_{i} \leq 1, i=1,2,3,4,5 ; x_{6} \geq 0 .
\end{array}
$$

$x^{\infty}=(0,1,0,1,1,20)$ is a minimizer with an objective value $f^{*}=-361.5$. We choose the initial point $x_{0}=$ $(1,1,1,1,1,10)$, iterate $=6$. 
Example 14 (see [11]). Consider

$$
\begin{array}{ll}
\min & f(x)=x_{1}^{2}+x_{2}^{2}+x_{3}^{2}+x_{4}^{2}-5 x_{1}-5 x_{2}-21 x_{3}-7 x_{4} \\
\mathrm{s.t.} & x_{1}^{2}+x_{2}^{2}+x_{3}^{2}+x_{4}^{2}+x_{1}-x_{2}+x_{3}-x_{4}-8 \leq 0 \\
& x_{1}^{2}+2 x_{2}^{2}+x_{3}^{2}+2 x_{4}^{2}+x_{1}-x_{4}-9 \leq 0 \\
& 2 x_{1}^{2}+x_{2}^{2}+x_{3}^{2}+2 x_{4}^{2}-x_{2}-x_{4}-5 \leq 0
\end{array}
$$

We choose the initial point $x_{0}=(1,1,1,1) \cdot x^{\infty}=$ $(0.2896,0.9150,2.1798,0.6265)$ is a minimizer with an objective value $f^{*}=-50.1192$, iterate $=40$.

\section{Acknowledgment}

This research was supported by the National Natural Science Foundation of China (no. 11271128).

\section{References}

[1] R. Fletcher and S. Leyffer, "Nonlinear programming without a penalty function," Mathematical Programming, vol. 91, no. 2, pp. 239-269, 2002.

[2] R. Fletcher, S. Leyffer, and P. L. Toint, "On the global convergence of a filter-SQP algorithm," SIAM Journal on Optimization, vol. 13, no. 1, pp. 44-59, 2002.

[3] R. Fletcher, N. I. M. Gould, S. Leyffer, P. L. Toint, and A. Wächter, "Global convergence of a trust-region SQP-filter algorithm for general nonlinear programming," SIAM Journal on Optimization, vol. 13, no. 3, pp. 635-659, 2002.

[4] C. C. Gonzaga, E. Karas, and M. Vanti, "A globally convergent filter method for nonlinear programming," SIAM Journal on Optimization, vol. 14, no. 3, pp. 646-669, 2003.

[5] A. Wächter and L. T. Biegler, "Line search filter methods for nonlinear programming: motivation and global convergence," SIAM Journal on Optimization, vol. 16, no. 1, pp. 1-31, 2005.

[6] A. Wächter and L. T. Biegler, "Line search filter methods for nonlinear programming: local convergence," SIAM Journal on Optimization, vol. 16, no. 1, pp. 32-48, 2005.

[7] C. M. Chin, "A global convergence theory of a filter line search method for nonlinear programming," Numerical Optimization Report, 2002.

[8] K. Su, "A globally and superlinearly convergent modified SQPfilter method," Journal of Global Optimization, vol. 41, no. 2, pp. 203-217, 2008.

[9] W. Wang, L.-S. Zhang, and Y.-F. Xu, "A revised conjugate gradient projection algorithm for inequality constrained optimizations," Journal of Computational Mathematics, vol. 23, no. 2, pp. 217-224, 2005.

[10] C. A. Floudas, P. M. Pardalos, C. S. Adjiman et al., Handbook of Test Problems in Local and Global Optimization, vol. 33, Kluwer Academic Publishers, Dordrecht, The Netherlands, 1999.

[11] K. Schittkowski, More Test Examples for Nonlinear Programming Codes, vol. 282, Springer, Berlin, Germany, 1987. 


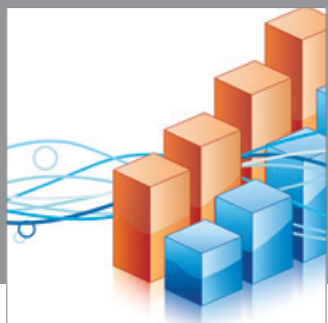

Advances in

Operations Research

mansans

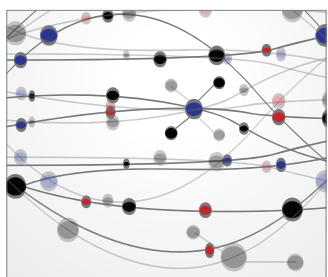

The Scientific World Journal
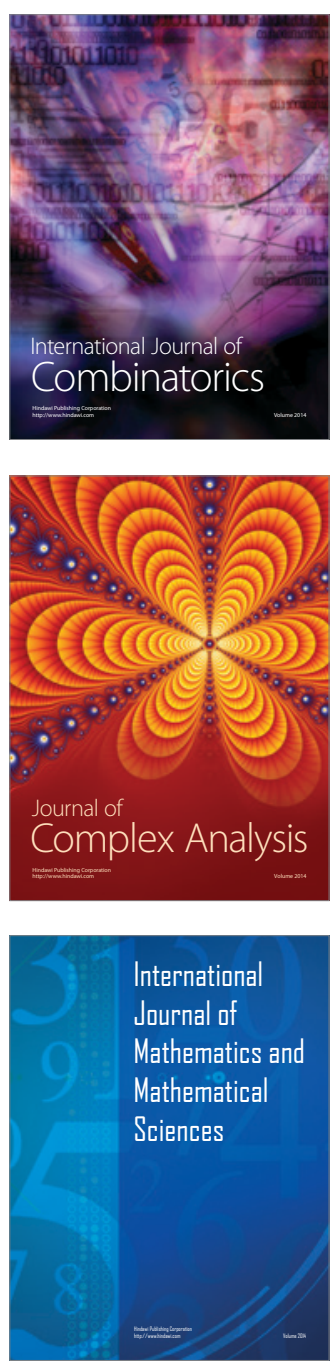
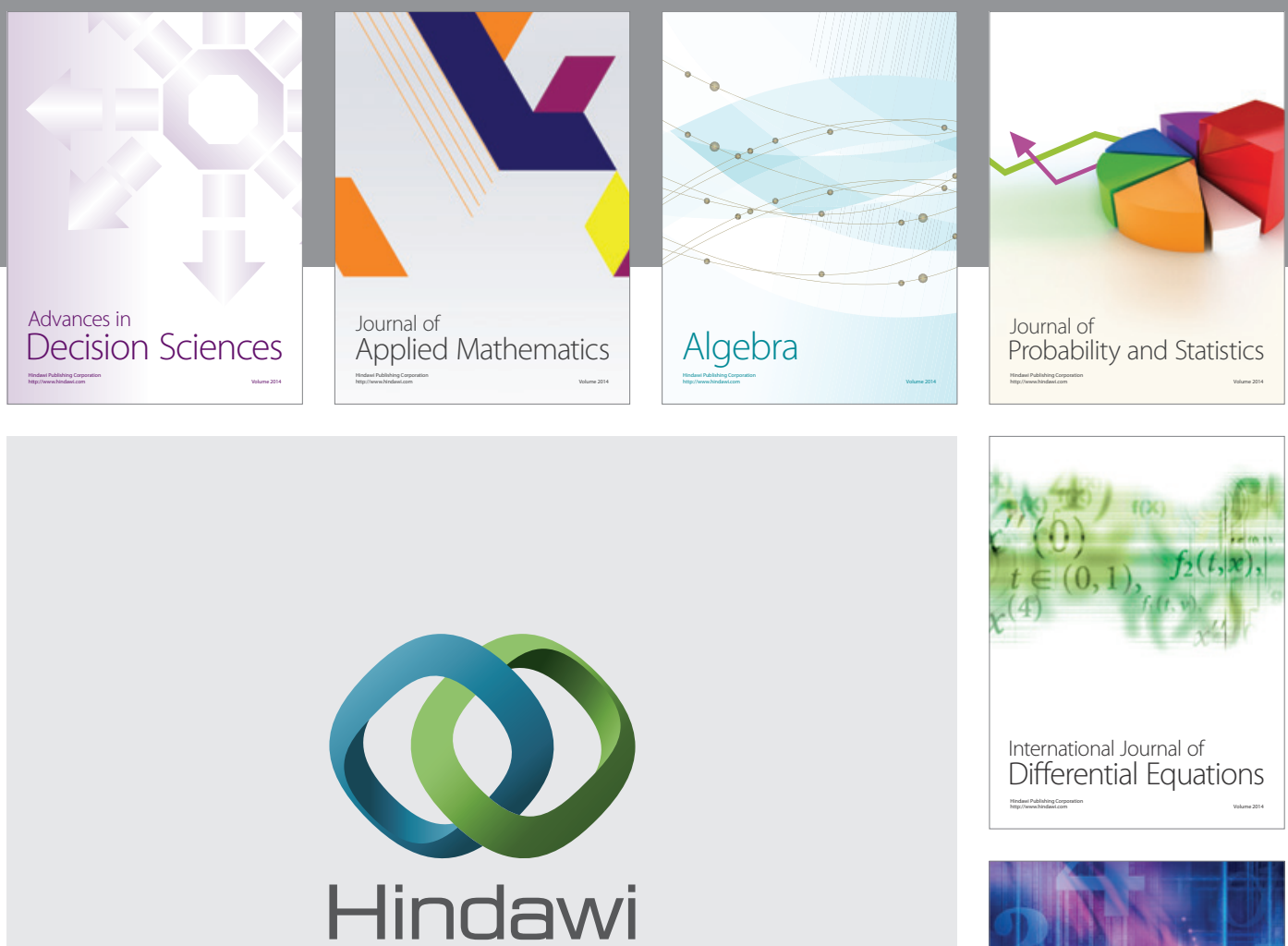

Submit your manuscripts at http://www.hindawi.com
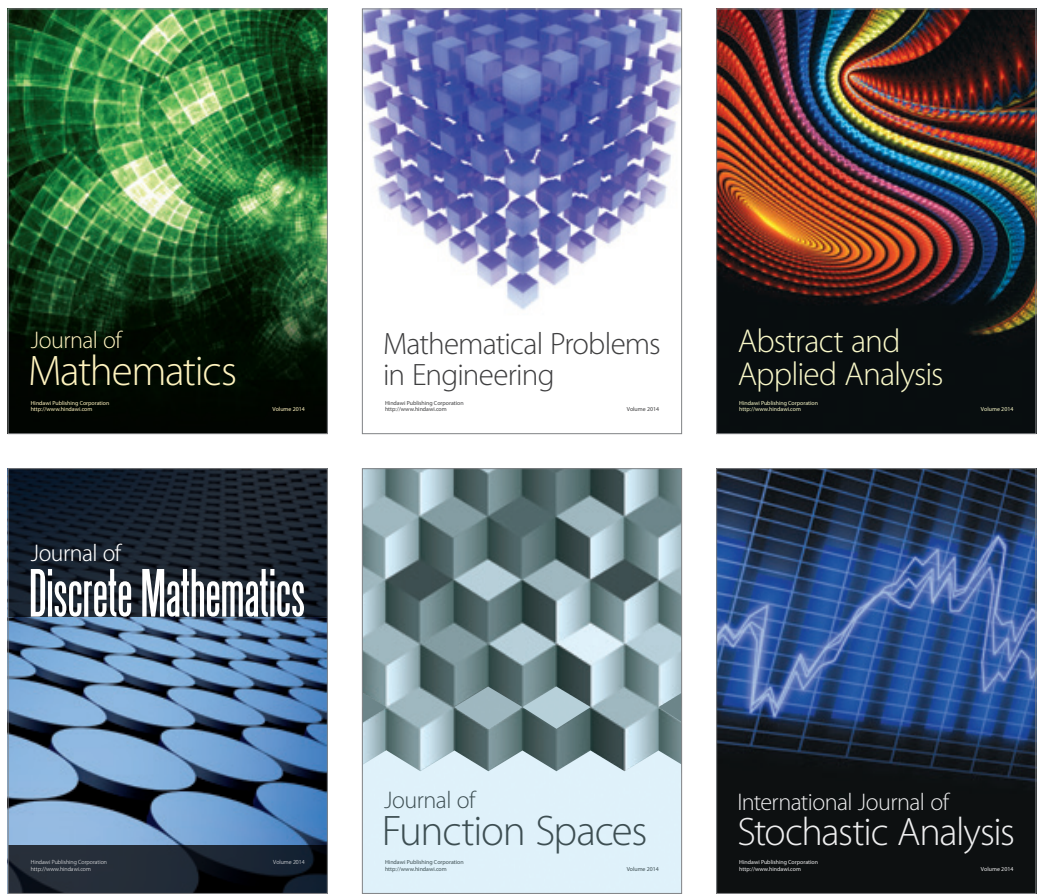

Journal of

Function Spaces

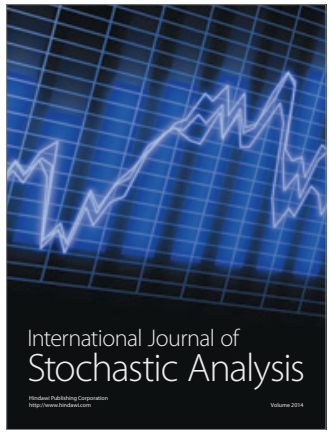

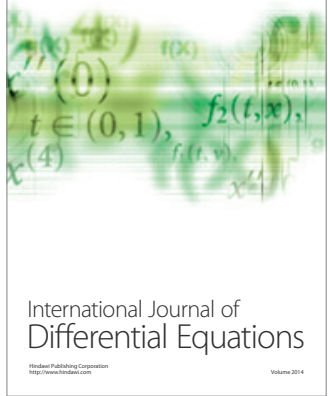
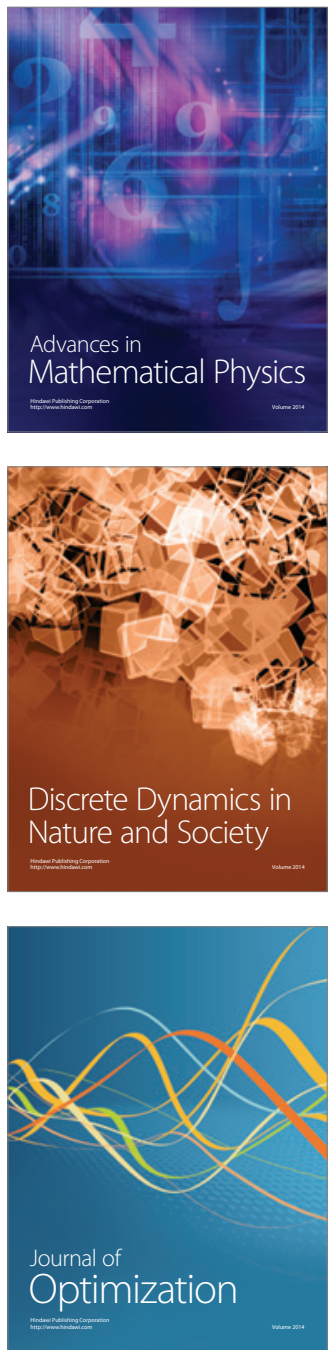